\title{
Japan lagging in influenza jabs
}

SIR - Influenza epidemics have high costs in morbidity, mortality and medical care. Vaccination of high-risk groups, such as the elderly or those with chronic illness, is the most effective measure for reducing the impact of the disease ${ }^{1}$. In the United States, the number of influenza immunizations has more than doubled in the past 10 years, and in 1994 coverage reached approximately $60 \%$ among people aged 65 and over ${ }^{2,3}$. Furthermore, the number of doses of vaccine distributed per 1,000 population more than doubled between 1990 and 1994 alone, as shown in the figure. The trend is similar to that seen in other developed countries, although several-fold differences are still seen in per capita vaccine distribution $^{4}$. In contrast, influenza vaccine distrib-

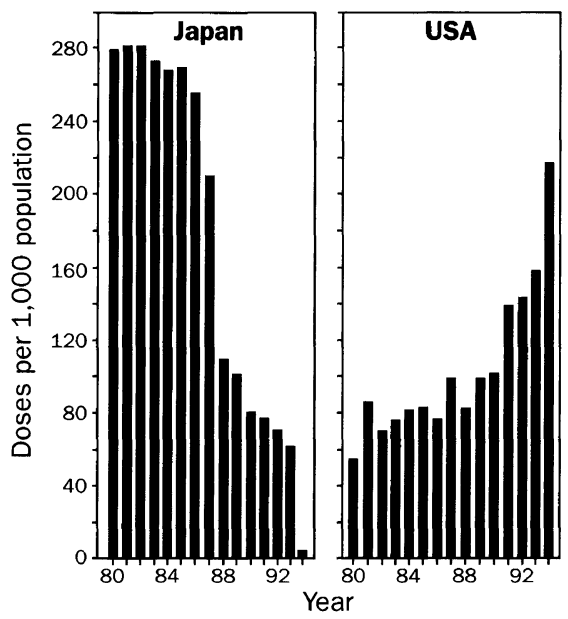

Influenza vaccine distribution in Japan and the United States, 1980-94, expressed as the number of doses distributed per 1,000 population. Japan's figure in 1994 is shown as the doses produced per 1,000 population, because accurate information on vaccine distribution is not available for that year.

ution in Japan has shown a marked decrease over the same period.

Influenza appeared on the list of targeted diseases in Japan from 1976 until June 1994. This policy was intended to control influenza epidemics in the entire community through suppressing transmission in schools ${ }^{5}$. Although the vaccination rate among schoolchildren was about $80 \%$ up to the early 1980 s, it showed a steep decline thereafter, to $18 \%$ in 1992 . This decline was due to poorly designed studies that alleged that the vaccine had little if any efficacy. These reports create scepticism about vaccination, not only among the general public but also among many medical professionals ${ }^{6}$

Influenza is considered a minor illness in Japan. There is no national recommendation of target groups for active immunization, nor any system for reimbursement. In this rapidly ageing society, very few older people volunteer for inoculation, and when they do they must pay approximately US\$50 to be vaccinated. Furthermore, Japanese pharmaceutical companies are decreasing or discontinuing their production of influenza vaccine. This contrasts strikingly with the United States where, since 1993, the federal government's Medicare programme has provided reimbursement for the cost of influenza vaccine and its administration ${ }^{2}$, and where the manufacturers have greatly expanded their production.

Many countries are developing comprehensive strategies to cope with the next influenza pandemic. Given recent experience, it seems unlikely that attitudes towards influenza and influenza vaccination in Japan will change in the near future. As a result, Japan may be ill prepared to join other countries in international efforts to confront the next pandemic.

Yoshio Hirota

Department of Public Health,

Faculty of Medicine 60,

Kyushu University,

Fukuoka 812-82, Japan

David S. Fedson

Pasteur Mérieux MSD,

3 Place Antonin Perrin,

F-69007 Lyon, France

Masaro Kaji

Kurume University,

Kurume, Fukuoka 830, Japan

1. C.D.C. Morbid. Mortal. Wkly Rep. 43 (RR-9), 1-13 (1994).

. Marwick, C. J. Am. med. Ass. 272, 1640-1642 (1994).

3. Marwick, C. J. Am. med. Ass. 273, 765 (1995).

4 Fedson, D. S. et al. Vaccine 13, 623-627 (1995)

5. Dowdle, W. R. et al. J. infect. Dis. 141, 258-264 (1980).

Hirota, Y. et al. Lancet 344, 408-409 (1994).

\section{Distrust of science}

SIR - Scientists complaining of the poor and declining image of themselves and of science itself have, following John Maddox's Commentary (Nature 378, 435-437; 1995) offer various remedies that scientists might exploit to improve the situation (Nature 379, 292; 1996). Each of these fails to take into account a much more deeprooted cause of the perceived malaise; a sea-change in the attitude of scientists themselves to the nature of science.

For a long period, from the midnineteenth century probably until the early 1960 s, science was driven on a great inductive wave and characterized by widespread acceptance of the notion that man, from observations of his surroundings, could derive universal laws, thereby conferring a degree of authority on science that had previously been the preserve of religion; and this at a time when religious authority was anyway in decline.

But for some time now, the perceived authority of inductive logic as the means of scientific advance has been in retreat: since The Logic of Scientific Discovery, scientists themselves have increasingly been encouraged to a more sceptical view and much of the scientific community now accepts many of Karl Popper's ideas on the deductive method of critically testing scientific ideas. This shift within the scientific community from a confident certainty to sceptical analysis of its theories is not simply a reaction to the well publicized failures of science; it is based on logically supported epistemological arguments, positively asserted by many scientists.

Much of the thrust of the discussion as to why the public distrusts science has centred on what is perceived to be an increasing chasm between scientists and the wider community. However, even though scientists may complain (with some justification) that many aspects of scientific endeavour are undervalued by the general public, they cannot ignore the fact that much public distrust is an accurate reflection of current scientific philosophy. It is not immediately obvious why the principle of healthy scepticism of scientific truths should be perceived as laudable within the scientific community but as unfortunate within the population at large.

Christopher B. Johnson

Department of Botany,

University of Reading,

Whiteknights, PO Box 221 ,

Reading RG6 6AS, UK

\section{Pensionable age?}

SIR - I am not surprised to hear Russian politicians criticizing the Soros scholarships for science and education. What does puzzle me is a similar reaction of scientists such as V. I. Rigin (Nature 378, 432; 1995). He believes that the scholarships awarded to Russian professors "exert a negative influence" on our educational system, as they go to old (meaning inefficient) hands and thus prevent them from being duly ousted. (I should perhaps say that I do not hold a Soros scholarship.) The same reasoning can apparently be applied to any outside financial help, whether regular or irregular.

No correlation between the number of scholarships and age of recipients is given, which alone makes Rigin's logic flimsy. More important is that there is no universal correlation between the age of a teacher and the quality of teaching. Some tribes have been known to regulate the average age of the population by withholding food from those considered a burden of the community. At what age does Rigin consider a professor ready to be disposed of?

\section{Yuri Berlin}

Shemyakin-Ovchinnikov Institute

of Bioorganic Chemistry,

Russian Academy of Sciences,

Moscow 117871, Russia 